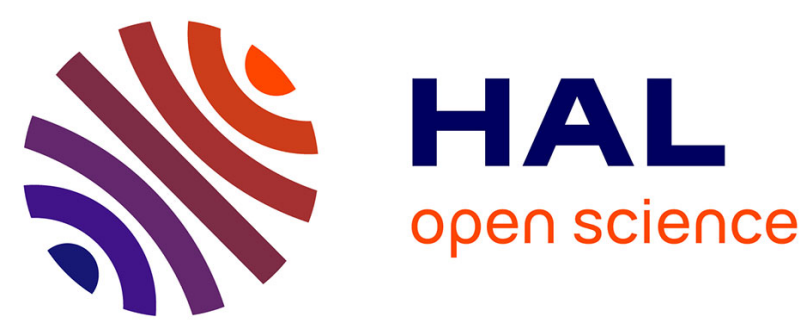

\title{
Susceptibility to Manipulation by Sincere Truncation: the Case of Scoring Rules and Scoring Runoff Systems
}

Eric Kamwa, Issofa Moyouwou

\section{To cite this version:}

Eric Kamwa, Issofa Moyouwou. Susceptibility to Manipulation by Sincere Truncation: the Case of Scoring Rules and Scoring Runoff Systems. Diss M.; Merlin V. Evaluating Voting Systems with Probability Models, Essays by and in honor of William Gehrlein and Dominique Lepelley, Springer Berlin Heidelberg, pp.275-295, 2021, Studies in Choice and Welfare, 10.1007/978-3-030-48598-6_12 . hal-02185965v2

\section{HAL Id: hal-02185965 \\ https://hal.univ-antilles.fr/hal-02185965v2}

Submitted on 13 Nov 2019

HAL is a multi-disciplinary open access archive for the deposit and dissemination of scientific research documents, whether they are published or not. The documents may come from teaching and research institutions in France or abroad, or from public or private research centers.
L'archive ouverte pluridisciplinaire HAL, est destinée au dépôt et à la diffusion de documents scientifiques de niveau recherche, publiés ou non, émanant des établissements d'enseignement et de recherche français ou étrangers, des laboratoires publics ou privés. 


\title{
Susceptibility to Manipulation by Sincere Truncation: The Case of Scoring Rules and Scoring Runoff Systems
}

Eric Kamwa and Issofa Moyouwou

\begin{abstract}
A voting rule is said to be vulnerable to the truncation paradox if some voters may seek to bring about a more preferable outcome by listing only a part of their sincere rankings on the competing candidates rather than listing their entire preference rankings on all the competing candidates. For three-candidate elections and for large electorates, under the Impartial Anonymous Culture assumption (IAC), this paper provides an evaluation of the likelihood of the truncation paradox occurring for the whole family of scoring rules and runoff scoring rules.
\end{abstract}

\section{Introduction}

During an election or a referendum, some people may choose not to vote. In case of a high level of abstention, the legitimacy of the results of an election may be challenged. The motivations of an abstainer may be dictated by various considerations among which strategic behavior plays a central role. It has been known since Doron and Kronick (1977) and Fishburn and Brams (1983) that a voter may do better to abstain than to vote since abstaining may result in the victory of a more preferable or desirable candidate. This counterintuitive voting event is known in the literature as the No-Show paradox. Following Nurmi (1999) and Felsenthal (2012), the few voting rules that are not vulnerable to the No-Show paradox include the Plurality rule, the Borda rule and Approval voting. ${ }^{1}$ According to Smith (1973), all the scoring

Eric Kamwa

LC2S UMR CNRS 8053, Université des Antilles, Faculté de Droit et d'Economie de la Martinique, F-97275 Schœlcher Cedex, e-mail: eric.kamwa@univ-antilles.fr

Issofa Moyouwou

Ecole Normale Supérieure-Department of Mathematics - University of Yaounde I, BP 47 Yaounde, Cameroon, e-mail: issofamoyouwou@ens.cm

${ }^{1}$ Under the Plurality rule, every voter casts one vote for only one candidate and the one with the greatest number of votes wins. With $m \geq 3$ candidates, the Borda rule gives $m-j$ points to a 
runoff systems are sensitive to the No-show paradox. With at least four candidates, Moulin (1988) showed that all the Condorcet consistent rules are also vulnerable to the No-Show paradox (see also Brandt et al., 2018, Duddy, 2013, Jimeno et al., 2009). A Condorcet consistent voting rule always elects the Condorcet winner when she exists. A Condorcet winner is a candidate who beats all the others in pairwise majority contests.

For voting situations with three candidates, Lepelley and Merlin (2001) computed the likelihood of the No-show paradox for three well-known scoring runoff rules; they concluded that when the electorate tends to infinity, the likelihood ${ }^{2}$ of the No-show paradox is equal to $2.14 \%$ for the Borda runoff, $5.40 \%$ for the Plurality runoff and $4.25 \%$ for the Antiplurality runoff. ${ }^{3}$ For their part, Kamwa et al. (2018) analyzed the No-show paradox for three-candidate elections with single-peaked preferences and found: $i$ ) in three-candidate elections with single-peaked preferences, all the scoring runoff rules located between the Borda runoff and the Antiplurality runoff are not sensitive to the No-show paradox; ii) single-peakedness of preferences greatly reduces the likelihood of the No-show paradox which nevertheless remains considerable.

It is perhaps to counter abstention behavior that several states (for instance, Bolivia, Belgium, Luxembourg and Romania) have decided to render voting compulsory. Notwithstanding compulsory voting, some voters may still manipulate the vote by using a weak version of abstention behavior called "sincere truncation". The sincere truncation of preferences, also called the truncation paradox, was first introduced in the social choice literature by Brams (1982). Let us assume a group of voters who are asked to rank (sincerely) a list of candidates from the most preferred to the least preferred and that voters are allowed to submit incomplete rankings; all the candidates not ranked or listed on a ballot are assumed to be less preferred than all those who are ranked (Fishburn and Brams, 1983, 1984). A voting rule is said to be vulnerable to sincere truncation if there are some configurations of ballots such that there is at least one voter who prefers the outcome obtained when he submits a sincere but incomplete ranking (truncated ranking) to the outcome obtained when he casts a complete sincere ranking.

A voting rule that is vulnerable to the No-Show paradox is also vulnerable to the truncation paradox, but the reverse is not necessary true (see Nurmi, 1999). Almost all the well-known voting rules are vulnerable to the truncation paradox. Fishburn and Brams (1984, p.402) showed that, as a consequence of Moulin's theorem, that all the Condorcet consistent rules are sensitive to the truncation paradox. For a nonexhaustive list of the voting rules vulnerable to the truncation paradox, the reader may refer to Felsenthal (2012), Nurmi (1999) and Fishburn and Brams (1984). The few exceptions are the Plurality rule, Plurality runoff and Approval voting.

candidate each time he is ranked $j$-th in a voter's ranking; the winner is the candidate with the largest total number of points. Under Approval voting, each voter can approve as many candidates as he wants. The winner is the candidate with the greatest number of approvals.

${ }^{2}$ Under the assumption of Impartial and Anonymous Culture (defined later).

${ }^{3}$ The Plurality runoff, the Borda runoff and the Antiplurality runoff will be defined later. 
Is the truncation paradox a rare oddity or a generalized behavior? To our knowledge, the only work that has tried to evaluate the likelihood of the truncation paradox is that of Plassmann and Tideman (1999); for three-candidate elections they focused on certain voting rules that include, amongst others, some Condorcet consistent rules, some scoring rules (Borda, Antiplurality) and some iterative scoring rules (iterative Plurality, iterative Antiplurality). They based their calculations on the spatial model for drawing voting situations. In this paper, we do the same job for the whole family of scoring rules and scoring runoff rules in three-candidate elections both under the universal and the single-peaked domains. Thus, we characterize all the paradoxical voting situations and then compute the exact likelihood of the paradox. We perform our analysis under the assumption of Impartial Anonymous Culture (IAC), which is one of the well-known assumptions often used for such a study. Under IAC, first introduced by Kuga and Hiroaki (1974) and later developed by Gehrlein and Fishburn (1976), each voting situation is assumed to be equally likely to occur. The likelihood of a given event is calculated with respect to the ratio between the number of voting situations in which the event is likely and the total number of possible voting situations. The number of voting situations associated with a given event can be reduced to the solutions of a finite system of linear constraints with (or without) rational coefficients. As recently pointed out in the social choice literature, when one is dealing with rational coefficients, the appropriate mathematical tools to find these solutions are Ehrhart polynomials. The background to this notion and its connection with the polytope theory can be found in Gehrlein and Lepelley $(2017,2011)$ and Lepelley et al. (2008). This technique has been widely used in numerous studies analyzing the probability of electoral events in the case of three-candidate elections under the IAC assumption. In this paper, we will follow the technique initiated by Cervone et al. (2005) for our computations. We say some few words on this technique in the appendices.

The rest of the paper is organized as follows: Section 2 is devoted to basic definitions. In Section 3, given a three-candidate election where voters have strict rankings, for all the one-shot and runoff scoring rules, we characterize all the voting situations vulnerable to the truncation paradox and then we compute the limiting probabilities. We do the same job in Section 4 by assuming that voters' preferences are single-peaked. Section 5 concludes.

\section{Notation and definitions}

\subsection{Preferences}

Let $N$ be a set of $n$ voters $(n \geq 2)$ and $A$ a set of $m$ candidates ( $m \geq 3$ ). Individual preferences are linear orders, these are complete, asymmetric and transitive binary relations on $A$. With $m$ candidates, there are exactly $m$ ! linear orders $P_{1}, P_{2}, \ldots, P_{m}$ ! on $A$. A voting situation is an $m$ !-tuple $\pi=\left(n_{1}, n_{2}, \ldots, n_{t}, \ldots, n_{m !}\right)$ that indicates the total number $n_{t}$ of voters casting each complete linear order $P_{t}, t=1,2, \ldots, m$ ! in 
such a way that $\sum_{t=1}^{m !} n_{t}=n$. In the sequel, we consider three candidates $a, b$ and $c$. In this case, we will simply write $a b c$ to denote the linear order on $A$ according to which $a$ is strictly preferred to $b, b$ is strictly preferred to $c$; and by transitivity $a$ is strictly preferred to $c$. Table 1 describes a voting situation with three candidates: there are six preference types and for $t=1,2, \ldots, 6, n_{t}$ is the total number of voters of type $t$.

Table 1 Voting situation and possible preference types with three candidates type 1: $a b c\left(n_{1}\right)$ type 3: bac $\left(n_{3}\right)$ type 5: $c a b\left(n_{5}\right)$ type 2: $a c b\left(n_{2}\right)$ type 4: $b c a\left(n_{4}\right)$ type $6: c b a\left(n_{6}\right)$

Given $a, b \in A$ and a voting situation $\pi$, we denote by $n_{a b}(\pi)$ (simply $n_{a b}$ ) the total number of voters who strictly prefer $a$ to $b$. If $n_{a b}>n_{b a}$, we say that $a$ majority dominates candidate $b$; or equivalently, $a$ beats $b$ in a pairwise majority voting. In such a case, we will simply write $a M(\pi) b$.

Possible actions of a voter include (i) ranking all candidates from the top-ranked candidate to the least preferred one; (ii) abstaining: no ranking is provided; or (iii) truncating: an incomplete ranking is provided. With the last action, it is assumed that all the candidates not ranked on a ballot are less preferred to all those who are ranked. With three candidates, when a voter truncates, he just states his most preferred candidate. For example with Table 1, if some voters of type 1 truncate, this leads to a new voting situation $\pi^{\prime}$ in which these voters only state $a--$ as their ranking. Note that when some voters truncate, this does not alter the size of the electorate as is the case when some voters abstain.

\subsection{Voting rules}

Scoring rules are voting systems that give points to candidates according to the position they have in voters' rankings. For a given scoring rule, the total number of points received by a candidate defines her score for this rule. The winner is the candidate with the highest score. In general, with $m \geq 3$ and complete strict rankings, a scoring vector is an $m$-tuple $w=\left(w_{1}, w_{2}, \ldots, w_{k}, \ldots, w_{m}\right)$ of real numbers such that $w_{1} \geq w_{2} \geq \ldots \geq w_{k} \geq \ldots \geq w_{m}$ and $w_{1}>w_{m}$. Given a voting situation $\pi$, each candidate receives $w_{k}$ each time she is ranked $k^{t h}$ by a voter. The score of a candidate $x \in A$ is the sum $S(\pi, w, x)=\sum_{t=1}^{m !} n_{t} w_{r(t, x)}$ where $r(t, x)$ is the rank of candidate $x$ according to voters of type $t$.

For uniqueness, we use the normalized form $\left(1, \frac{w_{2}-w_{m}}{w_{1}-w_{m}}, \ldots, \frac{w_{k}-w_{m}}{w_{1}-w_{m}}, \ldots, 0\right)$ of each scoring vector $w$. With three candidates, a normalized scoring vector has the shape $w_{\lambda}=(1, \lambda, 0)$ with $0 \leq \lambda \leq 1$. For $\lambda=0$, we obtain the Plurality rule; for $\lambda=1$, we have the Antiplurality rule and for $\lambda=\frac{1}{2}$, we get the Borda rule. From now on, we will denote by $S(\pi, \lambda, x)$, the score of candidate $x$ when the scoring vector is 
$w_{\lambda}=(1, \lambda, 0)$ and the voting situation is $\pi$. Table 2 gives the score of each candidate in $A=\{a, b, c\}$ given the voting situation of Table 1 .

Table 2 Scores with three candidates

\begin{tabular}{l}
$\overline{\overline{S(\pi, \lambda, a)}=n_{1}+n_{2}+\lambda\left(n_{3}+n_{5}\right)}$ \\
$S(\pi, \lambda, b)=n_{3}+n_{4}+\lambda\left(n_{1}+n_{6}\right)$ \\
$S(\pi, \lambda, c)=n_{5}+n_{6}+\lambda\left(n_{2}+n_{4}\right)$ \\
\hline
\end{tabular}

In one-shot voting, the winner is just the candidate with the largest score. Runoff systems involve two rounds of voting: at the first round, the candidate with the smallest score is eliminated; at the second round, a majority contest determines who is the winner.

With three candidates, when a voter of type 1 with the ranking $a b c$ truncates and submits $a--$, candidate $a$ receives 1 point in the new voting situation while both $b$ and $c$ receive zero points. Similar considerations hold for other types. Note that when some voters truncate, only the scores of candidates ranked second by some of these voters are affected and diminish. Moreover, truncation is only possible at the first round under runoff systems.

In our setting, we assume that ties among candidates will be broken alphabetically, e.g. $a$ wins all ties against other candidates; while $b$ wins all ties against $c$. Note that this special tie-breaking rule does not affect our results as we only deal with voting situations where the total number of voters tends to infinity. Let us now use an example in order to illustrate the truncation paradox for the voting rules we focus on.

\subsection{Illustrating the truncation paradox}

As stated above, among the scoring and the scoring runoff rules we focus on, only the Plurality rule and the Plurality runoff are not vulnerable to the truncation paradox. So, in our analysis of three-candidate elections, we will focus on $\lambda \in] 01$ ].

Now consider the following sincere voting situation $\pi$ with three candidates and 45 voters:

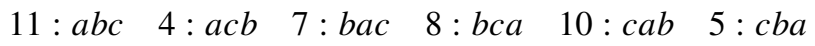

According to Table 2, the scores are as follows:

$$
S(\pi, \lambda, a)=15+17 \lambda ; \quad S(\pi, \lambda, b)=15+16 \lambda ; \quad S(\pi, \lambda, c)=15+12 \lambda
$$

It comes out that for all $\lambda \in] 01]$, we get $S(\pi, \lambda, a)>S(\pi, \lambda, b)>S(\pi, \lambda, c)$.

- The case of one-shot scoring rules. 
As $S(\pi, \lambda, a)>S(\pi, \lambda, b)>S(\pi, \lambda, c)$ for all $\lambda \in] 01]$, candidate $a$ is the winner. Assume that two voters with $b a c$ (type 3 ) truncate. Then, the new scores are:

$S\left(\pi^{\prime}, \lambda, a\right)=15+15 \lambda ; \quad S\left(\pi^{\prime}, \lambda, b\right)=15+16 \lambda ; \quad S\left(\pi^{\prime}, \lambda, c\right)=S(\pi, \lambda, c)=15+12 \lambda$

Candidate $a$ is no longer the winner since $S\left(\pi^{\prime}, \lambda, b\right)>S\left(\pi^{\prime}, \lambda, a\right)>S\left(\pi^{\prime}, \lambda, c\right)$; the new winner is candidate $b$. Since the two voters of type 3 benefit from the truncation, the truncation paradox can occur for all $\lambda \in] 01]$.

- The case of runoff scoring rules.

Given that $S(\pi, \lambda, a)>S(\pi, \lambda, b)>S(\pi, \lambda, c)$, candidate $c$ is eliminated at the first round. At the second round, candidate $a$ wins with $n_{a b}=25$ favorable votes against $n_{b a}=20$ votes in favor of $b$.

Assume that all the 5 voters with $c b a$ (type 6) truncate: they just state $c--$. In this case, the new scores are:

$$
S\left(\pi^{\prime}, \lambda, a\right)=15+17 \lambda ; \quad S\left(\pi^{\prime}, \lambda, b\right)=15+11 \lambda ; \quad S\left(\pi^{\prime}, \lambda, c\right)=15+12 \lambda
$$

For all $\lambda \in] 01]$, we get $S\left(\pi^{\prime}, \lambda, a\right)>S\left(\pi^{\prime}, \lambda, c\right)>S\left(\pi^{\prime}, \lambda, b\right)$ : candidate $b$ is eliminated at the first round. Since $n_{a c}=22$ and $n_{c a}=23, c$ wins the second round. So, by truncating their true preferences, the five voters of type 6 obtain a better outcome: the truncation paradox occurs.

\section{The vulnerability of scoring runoff rules to the truncation paradox in three-candidate elections}

Prior to the determination of the likelihood of the truncation paradox, we need to characterize all the voting situations under which this paradox is liable to occur.

\subsection{The case of one-shot scoring rules}

Consider a voting situation $\pi=\left(n_{1}, n_{2}, n_{3}, n_{4}, n_{5}, n_{6}\right)$ on $A=\{a, b, c\}$ and the one-shot rule with $0<\lambda \leq 1$. Let $\pi\left(\left[R_{j_{1}}, R_{j_{2}}, \ldots\right]\right)$ stands for the voting situation obtained from $\pi$ when all type $R_{j_{1}}, R_{j_{2}}, \ldots$ voters truncate their preferences. For example, $\pi[a b c]$ differs from $\pi$ only in the fact that at $\pi[a b c]$, candidate $a$ receives 1 point from each type 1 voter while the two other receive 0 points. Similarly, from $\pi$ to $\pi[a b c, a c b]$ the only change that occurs is that all type 1 voters and all type 2 voters now truncate their preferences to report $a \ldots$... For one-shot scoring rules, the following result identifies all voting situations in which the truncation paradox is possible. 
Proposition 1 Consider a voting situation $\pi=\left(n_{1}, n_{2}, n_{3}, n_{4}, n_{5}, n_{6}\right)$ on $A=\{a, b, c\}$, the one-shot rule associated with $0<\lambda \leq 1$ and a pair $\{x, y\}$ of candidates with $A \backslash\{x, y\}=\{z\}$.

If $x$ is the election winner at $\pi$, then the truncation paradox is liable to occur at $\pi$ in favor of $y$ if and only if $y$ is the election winner at $\pi([y x z, y z x])$.

Proof See Appendix A.

Remark 1 Given a voting situation with three candidates, only voters having the same top-ranked candidate can effectively benefit from truncating preferences by truly reporting their best candidate. For example, if $a$ is the winning outcome, it appears from Proposition 1 that truncating preferences may benefit either voters of type 3 and type 4 with $b a c$ and $b c a$ respectively; or else voters of type 5 and type 6 with the orderings $c a b$ and $c b a$ respectively. This is in contrast with other strategic misrepresentations of preferences which allow successful coordination among voters who may report a fake ranking with possibly a false best candidate - see Lepelley and Mbih (1994), Pritchard and Wilson (2007) or Mbih et al. (2009).

Proposition 2 Consider a one-shot scoring rule $F_{\lambda}, 0<\lambda \leq 1$. As the total number $n$ of voters tends to infinity, the limit probability of observing a voting situation in which the truncation paradox may occur is given by:

If $0<\lambda \leq \frac{1}{2}$,

$P_{T P}\left(F_{\lambda}\right)=\frac{\left(\begin{array}{c}10 \lambda^{14}-37 \lambda^{13}-179 \lambda^{12}+1310 \lambda^{11}-1778 \lambda^{10}-6319 \lambda^{9} \\ +26773 \lambda^{8}-25735 \lambda^{7}-67880 \lambda^{6}+259941 \lambda^{5}-408078 \lambda^{4} \\ +356643 \lambda^{3}-166536 \lambda^{2}+31833 \lambda\end{array}\right)}{6(3+\lambda)^{2}\left(3-2 \lambda+\lambda^{2}\right)^{2}(\lambda-2)^{2}(2 \lambda-3)^{2}(\lambda-1)(-3+5 \lambda)}$

If $\frac{1}{2} \leq \lambda \leq 1$,

$P_{T P}\left(F_{\lambda}\right)=\frac{\left(\begin{array}{c}2 \lambda^{13}+50 \lambda^{12}-194 \lambda^{11}-190 \lambda^{10}+2548 \lambda^{9}-5560 \lambda^{8} \\ -662 \lambda^{7}+26915 \lambda^{6}-62174 \lambda^{5}+73636 \lambda^{4}-48132 \lambda^{3} \\ +16425 \lambda^{2}-3564 \lambda+324\end{array}\right)}{12(3+\lambda)^{2}\left(3-2 \lambda+\lambda^{2}\right)^{2}(\lambda-2)^{2} \lambda^{2}(2 \lambda-3)}$

Proof See Appendix B for details of computations.

As the total number $n$ of voters tends to infinity, it appears from Proposition 2 that the limit probability, under the IAC assumption, of observing a voting situation in which the truncation paradox may occur given a one-shot scoring rule $F_{\lambda}$ increases from 0 to $\frac{3}{4}$ as the weight $\lambda$ increases from 0 (the Plurality rule) to 1 (the Antiplurality rule); for an overview of the behavior of $P_{\mathrm{TP}}\left(F_{\lambda}\right)$, see Figure 1 or Table 3 where we report some numerical evaluations. 
Fig. 1 Vulnerability of scoring rules to the truncation paradox

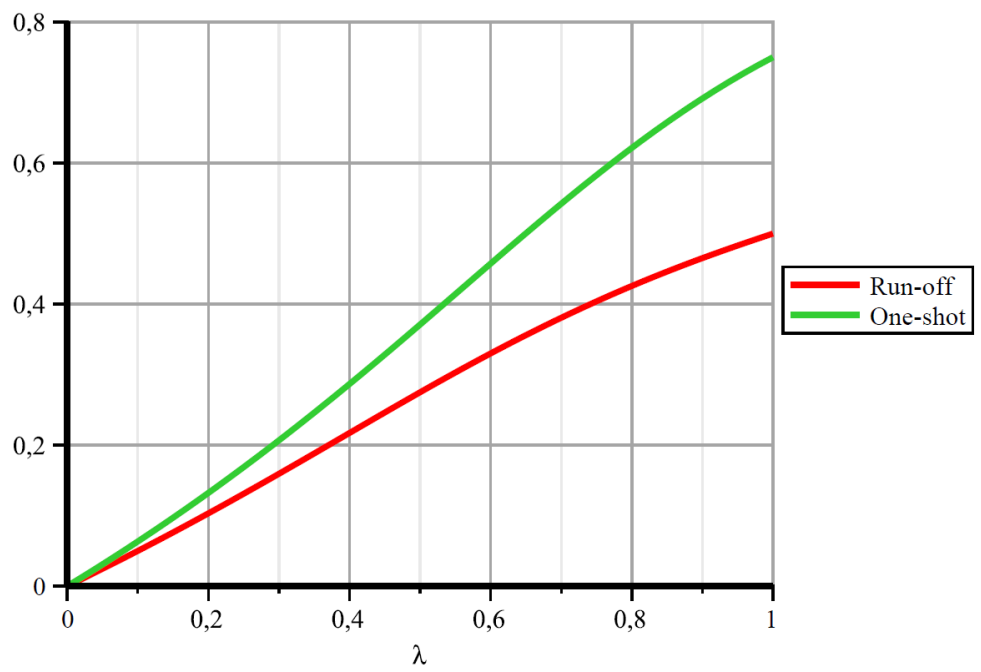

Table 3 Values of $P_{\mathrm{TP}}\left(F_{\lambda}\right)$ and $P_{\mathrm{TP}}\left(F_{\lambda}^{\prime}\right)$

\begin{tabular}{|c|c|c|c|c|c|c|c|c|c|c|c|}
\cline { 2 - 10 } \multicolumn{1}{c|}{} & 0 & 0.1 & 0.2 & 0.3 & 0.4 & 0.5 & 0.6 & 0.7 & 0.8 & 0.9 & 1 \\
\hline \hline$P_{\mathrm{TP}}\left(F_{\lambda}\right)$ & - & 0.06334 & 0.1322 & 0.2067 & 0.2866 & 0.3710 & 0.4575 & 0.5423 & 0.6215 & 0.6916 & 0.7500 \\
\hline$P_{\mathrm{TP}}\left(F_{\lambda}^{\prime}\right)$ & - & 0.0499 & 0.1032 & 0.1593 & 0.2172 & 0.2750 & 0.3300 & 0.3806 & 0.4257 & 0.4652 & 0.5000 \\
\hline
\end{tabular}

\subsection{The case of scoring runoff rules}

Consider the voting situation $\pi=\left(n_{1}, n_{2}, n_{3}, n_{4}, n_{5}, n_{6}\right)$ and a runoff rule with $0<$ $\lambda \leq 1$. Assume that at $\pi, z$ is eliminated at the first round and that $x$ wins against $y$ at the second round. For simplicity, we say that $x$ is the winner, $y$ is the challenger and $z$ is the (first-round) loser. To see how the truncation paradox arises under a runoff rule, recall that this paradox can be seen as a strategic behavior by some voters. Taking into account the specificity of runoff rules that combine both counting points at the first round and majority voting at the second round, successful truncations of preferences are either (i) in favor of the challenger when, by truncating their rankings, some voters make $x$ lose at the first round and cause the loser to be beaten by the challenger at the second round; or (ii) in favor of the loser who defeats the winner or the challenger in the second round. 
Proposition 3 Consider a voting situation $\pi=\left(n_{1}, n_{2}, n_{3}, n_{4}, n_{5}, n_{6}\right)$ on $A=\{a, b, c\}$ and a runoff rule with $0<\lambda \leq 1$. Assume that $x$ is the winner, $y$ is the challenger and $z$ is the first-round loser.

1. The truncation paradox is liable to occur at $\pi$ in favor of $y$ if and only if $y$ wins the majority duel against $z$ and $x$ is the first-round loser at $\pi$ ([yxz]).

2. The truncation paradox is liable to occur at $\pi$ in favor of $z$ if and only if $z$ wins the majority duel against $y$ and $x$ is the first-round loser at $\pi$ ([zxy]); or if $z$ wins the majority duel against $x$ and $y$ is the first-round loser at $\pi([z y x])$.

Proof See Appendix C.

In contrast with one-shot scoring rules, when the truncation paradox occurs under a runoff rule with three candidates, it is always reachable by a coalition of voters of the same type. Proposition 3 completely describes all the possible scenarios that support possible occurrence of the truncation paradox given a voting situation. These conditions lead us to some sets of linear constraints that characterize all possible occurrences of the truncation paradox under a runoff rule. Details are available in Appendix D. Computing the volume of all the corresponding polytopes leads to Proposition 4.

Proposition 4 Consider the scoring runoff rule $F_{\lambda}^{\prime}$ associated with the scoring vector $w_{\lambda}=(1, \lambda, 0)$ with $0<\lambda \leq 1$. As the total number $n$ of voters tends to infinity, the limit probability $P_{T P}\left(F_{\lambda}^{\prime}\right)$ of observing a voting situation in which the truncation paradox may occur is given by :If $0 \leq \lambda \leq \frac{1}{2}$,

$P_{T P}\left(F_{\lambda}^{\prime}\right)=-\frac{\left(\begin{array}{c}996096 \lambda^{20}-25010368 \lambda^{19}+286101152 \lambda^{18}-2000804220 \lambda^{17} \\ +9664972152 \lambda^{16}-34453144125 \lambda^{15}+94322255778 \lambda^{14} \\ -203353434975 \lambda^{13}+350716379871 \lambda^{12}-488312722095 \lambda^{11} \\ +551142449552 \lambda^{10}-504159008281 \lambda^{9}+372136194567 \lambda^{8} \\ -219653377992 \lambda^{7}+102140474607 \lambda^{6}-36558733185 \lambda^{5} \\ +9711109602 \lambda^{4}-1801641852 \lambda^{3}+208222083 \lambda^{2}-11278359 \lambda\end{array}\right)}{96(\lambda-1)^{2}(\lambda-2)^{2}(2 \lambda-3)^{2}(4 \lambda-3)^{2}(5 \lambda-3)^{2}\left(-2 \lambda+\lambda^{2}+3\right)\left(-5 \lambda+\lambda^{2}+3\right)^{2}\left(-4 \lambda+2 \lambda^{2}+3\right)\left(-7 \lambda+3 \lambda^{2}+3\right)}$

If $\frac{1}{2} \leq \lambda \leq 1$,

$P_{T P}\left(F_{\lambda}^{\prime}\right)=\frac{\left(\begin{array}{c}132 \lambda+9346 \lambda^{2}-55961 \lambda^{3}+161587 \lambda^{4}-283660 \lambda^{5} \\ +330502 \lambda^{6}-265921 \lambda^{7}+149437 \lambda^{8}-57766 \lambda^{9} \\ +14560 \lambda^{10}-2112 \lambda^{11}+128 \lambda^{12}-180\end{array}\right)}{288 \lambda^{3}(\lambda-2)^{2}(3-2 \lambda)\left(-2 \lambda+\lambda^{2}+3\right)\left(-4 \lambda+2 \lambda^{2}+3\right)}$

Proof See Appendix D for further details on the computation.

The limit, as the total number $\mathrm{n}$ of voters tends to infinity, and under the IAC assumption, of the probability of observing a voting situation in which the truncation paradox may occur given the runoff scoring rule $F_{\lambda}^{\prime}$ increases from 0 to 0.5 as the weight $\lambda$ increases from 0 (the Plurality runoff rule) to 1 (the Antiplurality runoff rule). Moreover each one-shot scoring rule is more vulnerable to the truncation 
paradox than its corresponding runoff version. For an overview of the behavior of $P_{\mathrm{TP}}\left(F_{\lambda}^{\prime}\right)$, see Figure 1 or Table 3 where some numerical evaluations are reported. Finally, while the Plurality rule is not vulnerable to the truncation paradox as a oneshot rule or a runoff rule, the Antiplurality rule appears to be the most vulnerable rule among both the one-shot and runoff scoring rules with three candidates.

An analysis of the vulnerability of runoff scoring rules to profitable abstention in three-candidate elections is available from Lepelley and Merlin (2001) for the main scoring runoff rules, and from Kamwa et al. (2018) for the whole family of scoring runoff rules. In Table 4, we report the limiting probabilities of the no-show paradox obtained by Lepelley and Merlin (2001) for the universal and by Kamwa et al. (2018) for the single-peaked domain.

Table 4 Limiting probabilities of the No-show paradox under the Universal and the single-peaked domains for scoring runoff rules

\begin{tabular}{|c|c|c|c|c|c|c|c|c|c|c|c|}
\hline \hline & \multicolumn{10}{|c|}{$\lambda$} \\
\hline & 0 & 0.1 & 0.2 & 0.3 & 0.4 & 0.5 & 0.6 & 0.7 & 0.8 & 0.9 & 1 \\
\hline Universal & 0.0408 & 0.0382 & 0.0351 & 0.0313 & 0.0272 & 0.0243 & 0.0263 & 0.0299 & 0.0341 & 0.0383 & 0.0425 \\
\hline Single-peaked & 0.0278 & 0.0208 & 0.0136 & 0.0067 & 0.0016 & 0 & 0 & 0 & 0 & 0 & 0 \\
\hline \hline
\end{tabular}

Comparing the probabilities by Lepelley and Merlin (2001) and Kamwa et al. (2018) on the No-show paradox with those we obtain on the truncation paradox, it emerges that the truncation paradox is always more likely to occur than the No-show paradox for all $0<\lambda \leq 1$. This is consistent with the fact that the truncation paradox is a weak version of the No-show paradox.

\section{The impact of single-peaked preferences}

Kamwa et al. (2018) showed that when preferences are single-peaked in threecandidate elections, the No-show paradox never occurs with all the scoring runoff rules located between the Borda runoff and the Antiplurality runoff, i.e., for all $\lambda \in\left[\begin{array}{ll}\frac{1}{2} & 1\end{array}\right]$. It emerges from their probability computations that the likelihood of the No-show paradox is drastically reduced with single-peaked preferences. In this section, we also want to check what happens with the truncation paradox when preferences are single-peaked.

With three candidates, when preferences are single-peaked, there is one candidate that is not bottom ranked. On $A=\{a, b, c\}$, we assume without loss of generality that candidate $c$ is never bottom ranked. Table 5 describes a voting situation with three candidates and single-peaked preferences.

In the sequel we assume that only the four preference types in Table 5 are observable. Note that when candidate $a$ wins in a voting situation, only voters of type 4 and 
Table 5 Single-peaked preferences and scores on $A=\{a, b, c\}$

\begin{tabular}{ll}
\hline \multicolumn{2}{c}{ Preference types } \\
\hline$n_{2}: a c b$ & $n_{4}: b c a$ \\
$n_{5}: c a b$ & $n_{6}: c b a$ \\
\hline
\end{tabular}

\begin{tabular}{c}
\hline Scores at the first round \\
\hline$S(\pi, \lambda, a)=n_{2}+\lambda n_{5}$ \\
$S(\pi, \lambda, b)=n_{4}+\lambda n_{6}$ \\
$S(\pi, \lambda, c)=n_{5}+n_{6}+\lambda\left(n_{2}+n_{4}\right)$ \\
\hline
\end{tabular}

6 who strictly prefer $b$ to $a$ may truncate their rankings in order to favor the election of $b$. Since candidate $a$ is bottom ranked by all those voters, there is no way left to favor candidate $b$ by preference truncation. Similarly, when candidate $b$ wins under a one-shot scoring rule, there is no opportunity to favor candidate $a$ by preference truncation. The conditions of Proposition 1 for one-shot scoring rules still apply for viable sincere truncation of preference when preferences are single-peaked, except for the restriction just outlined and reported in Proposition 5.

Proposition 5 Consider a voting situation on $A=\{a, b, c\}$ with single-peaked preferences such that candidate $c$ is never bottom ranked in the individual preferences. Assume that the voting rule is a one-shot scoring rule.

- When candidate a (or candidate $b$ ) is the election winner in $\pi$ for $\lambda \in] 01[$, then the truncation paradox is liable to occur only in favor of $c$.

- When candidate $c$ is the election winner in $\pi$ for $\lambda \in] 01$ ], it is possible to favor a candidate in $A \backslash\{c\}$ by sincere truncation of preferences.

Proof See Appendix E.

In the same way, the conditions of Proposition 3 also identify all the scenarios in which a runoff rule is vulnerable to the truncation paradox when individual preferences are single-peaked except for the restriction provided in Proposition 6.

Proposition 6 Consider a voting situation on $A=\{a, b, c\}$ with single-peaked preferences such that candidate $c$ is never bottom ranked in individual preferences.

i) Assume that candidate c is eliminated after the first run. In this case, the truncation paradox can occur only in favor of candidate c; and only for all the scoring runoff rules associated with $\lambda \in] 0, \frac{1}{2}[$.

ii) Assume that candidate $a$ or $b$ wins the second run versus candidate $c$. The truncation paradox never occurs for all the scoring runoff rules with $\lambda \in] 0,1]$.

iii) Assume that candidate $c$ wins the second run versus candidate $a$ or $b$. The truncation paradox can occur for all the scoring runoff rules such that $\lambda \in] 0,1[$.

Proof See Appendix F.

What emerges from Proposition 6 is that single-peaked preferences do not vitiate the truncation paradox in the same manner as they do with the No-show paradox; as with the No-show paradox, they totally obviate the truncation paradox for voting situations under which the never-bottom-ranked candidate loses at the second stage. 
For three-candidate elections with single-peaked preferences, Table 6 gives all the scoring runoff rules vulnerable to the truncation paradox and to the No-show paradox for all the possible configurations: i) $a$ wins the second stage versus $b$; ii) $a$ or $b$ wins the second stage versus $c$; and iii) $c$ wins the second stage versus $a$ or $b$. The reader can then see from Table 6 that the impact of single-peaked preferences on the truncation paradox is not the same as on the Abstention paradox although the first paradox is the weaker version of the second.

Table 6 Vulnerable scoring runoff rules with three candidates and single-peaked preferences

\begin{tabular}{|c|c|c|c|}
\cline { 2 - 4 } Second-round opponents & $a v s . b$ & $a$ or $b$ wins $v s . c$ & $c$ wins $v s . a$ or $b$ \\
\hline Abstention $^{\star}$ & $\lambda \in\left[0 \frac{1}{2}[\right.$ & - & $\lambda \in] 0 \frac{1}{2}[$ \\
\hline Truncation & $\lambda \in] 0 \frac{1}{2}[$ & - & $\lambda \in] 01[$ \\
\hline
\end{tabular}

$\star$ From Kamwa et al. (2018)

Further aspects of the behavior of one-shot scoring rules are perhaps more interesting. Note that in the general case, the probability that a one-shot scoring rule exhibits the truncation paradox, given that the winner is a given candidate, is the same from one candidate to another. But when preferences are single-peaked, results from computations provided for the next proposition and sketched in Figure 2 show that a one-shot scoring rule is more vulnerable to the truncation paradox when the centrist candidate $c$ is elected than when the leftist candidate $a$ or the rightist candidate $b$ is elected. 
Fig. 2 Single-peakedness: vulnerability of one-shot rules to the truncation paradox

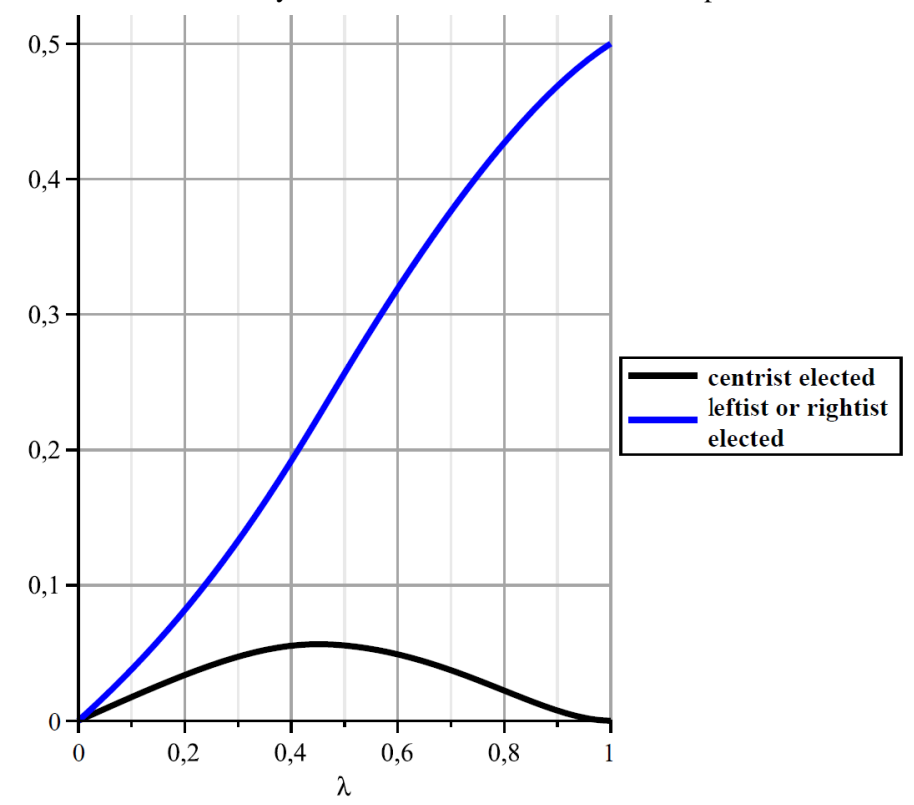

In the next propositions, we report global probabilities we obtained by performing the probability computation over all the possible scenarios for the truncation paradox on the single-peaked domain. As expected, these probabilities are lower than those we observe on the universal domain. However, these probabilities remain significantly high.

Proposition 7 Consider the one-shot rule associated with the scoring vector $w_{\lambda}=$ $(1, \lambda, 0)$ with $0<\lambda \leq 1$. As the total number $n$ of voters tends to infinity, the limit probability $P_{T P}\left(F_{\lambda}, S P\right)$ of observing a voting situation in which the truncation paradox may occur is as follows:

$$
\begin{aligned}
& \text { If } 0 \leq \lambda \leq \frac{1}{2}, P_{T P}\left(F_{\lambda}, S P\right)=\frac{\lambda\left(-94 \lambda^{5}-441 \lambda^{4}-30 \lambda^{7}+108 \lambda^{6}+1602 \lambda^{3}-2456 \lambda^{2}+1812 \lambda-513+4 \lambda^{8}\right)}{9(2 \lambda-3)(\lambda-1)^{2}(-2+\lambda)^{2}\left(3+\lambda^{2}-2 \lambda\right)(\lambda+3)} \\
& \text { If } \frac{1}{2} \leq \lambda \leq 1, P_{T P}\left(F_{\lambda}, S P\right)=\frac{-65 \lambda^{2}-6 \lambda^{4}+38 \lambda^{3}+43 \lambda-4 \lambda^{5}+2 \lambda^{6}-4}{(\lambda-2)^{2}(\lambda+3)\left(\lambda^{2}-2 \lambda+3\right)}
\end{aligned}
$$

Proposition 8 Consider the runoff rule associated with the scoring vector $w_{\lambda}=$ $(1, \lambda, 0)$ with $0<\lambda \leq 1$. As the total number $n$ of voters tends to infinity, the limit probability $P_{T P}\left(F_{\lambda}^{\prime}, S P\right)$ of observing a voting situation in which the truncation paradox may occur is as follows: 
If $0 \leq \lambda \leq \frac{1}{2}, P_{T P}\left(F_{\lambda}^{\prime}, S P\right)=\frac{\lambda\left(-110 \lambda^{6}+322 \lambda^{5}-64 \lambda^{7}+4374 \lambda^{2}+365 \lambda^{4}+22 \lambda^{8}+657-2684 \lambda^{3}-2850 \lambda\right)}{24(2-\lambda)(-3+\lambda)(-3+2 \lambda)(\lambda-1)^{2}\left(3+\lambda^{2}-2 \lambda\right)(\lambda+3)}$

If $\frac{1}{2} \leq \lambda \leq 1, P_{T P}\left(F_{\lambda}^{\prime}, S P\right)=\frac{(\lambda-1)^{2}\left(-\lambda^{3}+2 \lambda^{2}+7 \lambda-15+\lambda^{4}\right)}{4(2-\lambda)\left(\lambda^{2}-2 \lambda+3\right)(-3+2 \lambda)(\lambda+3)}$

The vulnerabilities to the truncation paradox reported in Proposition 7 and 8 are computed using very similar arguments to the proofs of Proposition 3 and Proposition 4 respectively. One simply needs to consider the possible scenarios described in Proposition 5 and Proposition 6; the details are omitted.

Table 7 reports the figures we get from Propositions 7 and 8 .

Table 7 Values of $P_{\mathrm{TP}}\left(F_{\lambda}, S P\right)$ and $P_{\mathrm{TP}}\left(F_{\lambda}^{\prime}, S P\right)$

\begin{tabular}{c|c|c|c|c|c|c|c|c|c|c|c|}
\cline { 2 - 10 }$\lambda$ & 0 & 0.1 & 0.2 & 0.3 & 0.4 & 0.5 & 0.6 & 0.7 & 0.8 & 0.9 & 1 \\
\hline \hline$P_{\mathrm{TP}}\left(F_{\lambda}, S P\right)$ & - & 0.0553 & 0.1155 & 0.1801 & 0.2472 & 0.3122 & 0.3683 & 0.4137 & 0.4489 & 0.4762 & 0.5000 \\
\hline$P_{\mathrm{TP}}\left(F_{\lambda}^{\prime}, S P\right)$ & 0 & 0.0158 & 0.0284 & 0.0357 & 0.0361 & 0.0293 & 0.0207 & 0.0129 & 0.0063 & 0.0017 & 0 \\
\hline \hline
\end{tabular}

With runoff scoring rules the effect of single-peaked preferences is indeed remarkable: it reduces the probability of truncation to less than 0.035 for all the runoff scoring rules. Moreover, when the centrist candidate $c$ is the winner, the probability that a runoff scoring rule is very low and even null when the weight $\lambda$ lies between 0.5 and 1 . What is also surprising is that the runoff version of the Antiplurality rule is now immune to the truncation paradox. To see this, note that when $\lambda=1$ and candidate $c$ is ranked last by no voter, candidate is always qualified for the second round whether preferences are truncated by voters of type 2 (or type 4 ) or not. Figure 3 shows how single-peakedness imposes a downwards curve on the vulnerability of runoff scoring rules to the truncation paradox. 
Fig. 3 Single-peakedness: vulnerability of runoff rules to the truncation paradox

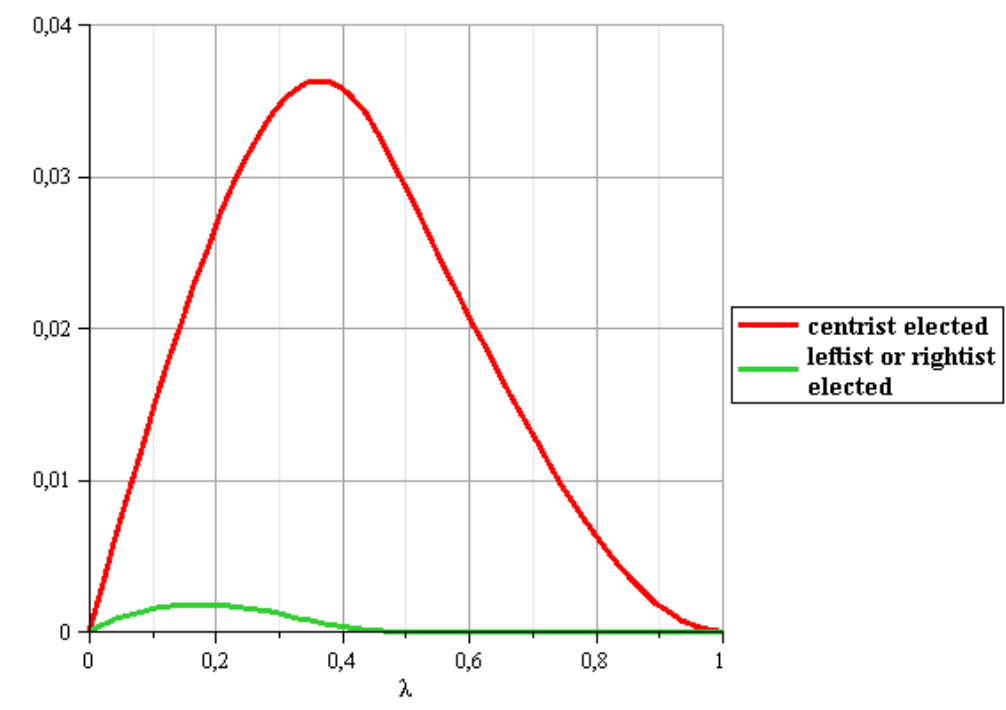

Figure 4 is a comparative visualization of the vulnerability of both one-shot scoring rules and runoff scoring rules to the truncation paradox when preferences are single-peaked. It obviously highlights the fact that each one-shot scoring rule is still more vulnerable to the truncation paradox than its runoff version, even with single-peaked preferences. 
Fig. 4 Single-peakedness: vulnerability of one-shot rules and runoff rules to the truncation paradox

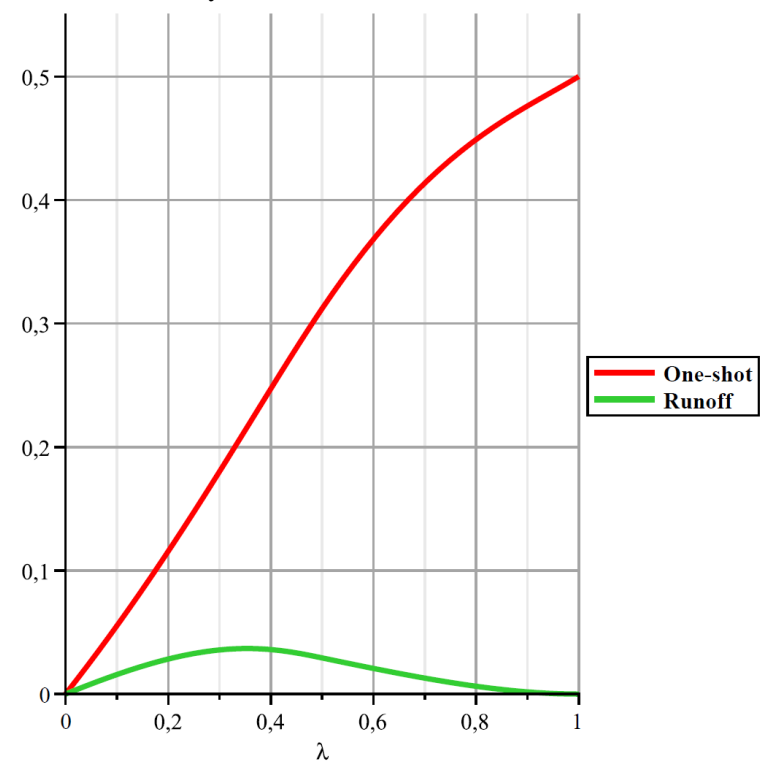

\section{Concluding remarks}

Since Fishburn and Brams (1984), we have known that almost all the well-known voting rules are vulnerable to the truncation paradox except the Plurality rule, Plurality runoff and Approval voting. In this paper, we have characterized all the threecandidate voting situations under which the truncation paradox can occur for scoring rules and scoring runoff rules under both the universal and the single-peaked domains. Then we computed the limiting probability of the truncation paradox. By comparing our results to those obtained by Lepelley and Merlin (2001) and Kamwa et al. (2018) concerning the likelihood of the Abstention paradox, we concluded that the Abstention paradox is less likely to occur than the truncation paradox. Hence, making voting compulsory in order to counter the paradoxical outcomes caused by abstention behavior seems not to be a good choice at all.

With single-peaked preferences, we found that the occurrence of the truncation paradox depends on the configuration of the second run: if the never-bottom ranked candidate loses the second run versus one of the other candidates, the truncation paradox never occurs; if this candidate is rushed out at the first stage, the truncation paradox never occurs with all the scoring runoff rules located between the Borda runoff and the Antiplurality runoff.

Acknowledgements The authors are grateful to two anonymous reviewers for their helpful remarks and comments. 


\section{Appendices}

\section{A. Proof of Proposition 1}

Consider a voting situation $\pi=\left(n_{1}, n_{2}, n_{3}, n_{4}, n_{5}, n_{6}\right)$ on $A=\{a, b, c\}$, the one-shot rule associated with $0<\lambda \leq 1$ and a pair $\{x, y\}$ of candidates. Let $z$ be the third candidate.

Necessity. Assume that $x$ is the election winner at $\pi$, and that the truncation paradox is liable to occur in $\pi$ in favor of $y$. Then by truncating their true preferences, a coalition of voters, say $S$, favors the election of $y$. Moreover each voter in $S$ strictly prefers $y$ to $x$. Since the truncation operation only affects the second-ranked candidates of each voter in $S$, then the preferences of each voter in $S$ is $y x z$ or $y z x$. At the new voting situation $\pi^{\prime}, y$ wins. Without loss of generality, we denote by $n_{x y z}(\pi)$ the total number of voters in $\pi$ who rank $x$ first, $y$ second and $z$ last at $\pi$. Note that $|S| \leq n_{y x z}(\pi)+n_{y z x}(\pi)$. Then from $\pi^{\prime}$ to $\pi([y x z])$, the score of $y$ increases, the scores of both $x$ and $z$ decrease. Hence $y$ also wins in $\pi([y x z, y z x])$.

Sufficiency. Assume that $x$ is the election winner at $\pi$ while $y$ wins in $\pi$ ([yxz,yzx]). Clearly, the truncation paradox is liable to occur in $\pi$ in favor of $y$ since all voters who truncate their preferences in $\pi([y x z, y z x])$ prefers $y$ to $x$.

\section{B. Computation details for Proposition 2}

Let $T_{x}$ denote the set of all voting situations in which $x$ is the election winner while the truncation paradox is liable to occur; and $T_{x y}$ the subset of $T_{x}$ that consists of all voting situations in which truncating preferences may favor the election of $y$. Note for example that

$$
T_{a}=T_{a b} \cup T_{a c} \text { and }\left|T_{a}\right|=\left|T_{a b}\right|+\left|T_{a c}\right|-\left|T_{a b} \cap T_{a c}\right| .
$$

By Proposition $1, \pi \in T_{a b}$ if and only if $S(\pi, \lambda, a) \geq S(\pi, \lambda, b), S(\pi, \lambda, a) \geq$ $S(\pi, \lambda, c), S(\pi[b a c, b c a], \lambda, b)>S(\pi[b a c, b c a], \lambda, a)$ and $S(\pi[b a c, b c a], \lambda, b) \geq$ $S(\pi[b a c, b c a], \lambda, c)$. Equivalently,

$$
\pi \in T_{a b} \Longleftrightarrow\left\{\begin{array}{l}
(\lambda-1) n_{1}-n_{2}+(1-\lambda) n_{3}+n_{4}-\lambda n_{5}+\lambda n_{6} \leq 0 \\
-n_{1}+(\lambda-1) n_{2}-\lambda n_{3}+\lambda n_{4}+(1-\lambda) n_{5}+n_{6} \leq 0 \\
(1-\lambda) n_{1}+n_{2}-n_{3}-n_{4}+\lambda n_{5}-\lambda n_{6}<0 \\
-\lambda n_{1}+\lambda n_{2}-n_{3}-n_{4}+n_{5}+(1-\lambda) n_{6} \leq 0
\end{array}\right.
$$

Clearly, each of the six possible sets $T_{x y}$ with $x, y \in A$ can be similarly described by a set of four linear constraints as with $T_{a b}$ above. As $n$ tends to infinity, vol $\left(P_{x y}\right)$ is the 5 -dimensional volume of the polytope $P_{x y}$ obtained from the characterization of $T_{x y}$ by replacing each $n_{j}$ by $p_{j}=\frac{n_{j}}{n}$. Note that some inequalities in the characterization of $P_{x y}$ may be strict. We simply ignore this while evaluating vol $\left(P_{x y}\right)$ by considering 
the closure of $P_{x y}$ obtained from the characterization of $P_{x y}$ by turning each strict inequality $(<)$ to its larger form $(\leq)$; by doing so, we simply move from $P_{x y}$ to its closure without changing the volume. Taking into account that $T_{a}, T_{b}$ and $T_{c}$ are disjoint sets of voting situations, and since by symmetries, all the six possible $T_{x y}$ generates polytopes of equal volume, the limit probability $P\left(F_{\lambda}, T P, I A C\right)$ under the IAC assumption, of observing a voting situation in which the truncation paradox may occur is

$$
P_{\mathrm{TP}}(F)=\frac{\operatorname{vol}\left(P_{a}\right)+\operatorname{vol}\left(P_{b}\right)+\operatorname{vol}\left(P_{c}\right)}{\operatorname{vol}(P)}=720 \operatorname{vol}\left(P_{a b}\right)-360 \operatorname{vol}\left(P_{a b} \cap P_{a c}\right)
$$

where $P$ is the simplex $P=\left\{\left(p_{1}, p_{2}, \ldots, p_{6}\right): \sum_{t=1}^{6} p_{j}=1\right.$ with $p_{j} \geq 0, j=$ $1,2, \ldots, 6\}$. Given $0<\lambda \leq 1$, computing $\operatorname{vol}\left(P_{a b}\right)$ and $\operatorname{vol}\left(P_{a b} \cap P_{a c}\right)$, one obtains the result of Proposition 1. All volume computations performed in this paper use the same technique as in Cervone et al. (2005). ${ }^{4}$ Roughly, one needs for example to determine all vertices of the given polytope and then triangulate the set of those vertices into simplices. More details are presented in Moyouwou and Tchantcho (2015) and Gehrlein and Lepelley (2011); further illustrations are available in Gehrlein et al. (2015) or more recently in Lepelley et al. (2018). A Maple procedure is also available from authors upon request. Of course, there is an abundant literature on volume computations with very efficient algorithms and packages such as Büeler et al. (2000) and Lawrence (1991) for Maple users or Bruns and Ichim (2010) and Bruns et al. $(2019,2018)$.

\section{Proof of Proposition 3}

Consider a voting situation $\pi=\left(n_{1}, n_{2}, n_{3}, n_{4}, n_{5}, n_{6}\right)$ on $A=\{a, b, c\}$ and the runoff rule associated with $0<\lambda \leq 1$. Assume that $x$ is the winner, $y$ is the challenger and $z$ is the first-round loser.

1. Necessity. First assume that the truncation paradox is liable to occur at $\pi$ in favor of $y$. Then by truncating their true preferences, a coalition of voters, say $S$, diminishes the score of $x$ in such a way that $x$ is now ruled out at the first round and $y$ wins against $z$ at the second round. Each voter in $S$ strictly prefers $y$ to $x$. The truncation operation by such a voter is only intended to diminish the score of $x$ at the first round. Thus the preferences of each voter in $S$ is $y x z$. In the new voting situation $\pi^{\prime}, y$ wins. Since from $\pi^{\prime}$ to $\pi([y x z])$, the score of $y$ does not decrease, the score of $x$ does not increase, the score of $z$ is unchanged and the second round duel is not affected by the truncation operation, then $y$ also wins in $\pi([y x z])$ against $z$ at the second round.

${ }^{4}$ This technique has recently been used in many research papers, such as Diss and Gehrlein (2015, 2012), Gehrlein et al. (2015), Kamwa et al. (2018), Kamwa and Valognes (2017), Moyouwou and Tchantcho (2015) and Kamwa (2019) among others. 
Sufficiency. Assume that $y$ wins the majority duel against $z$ and $x$ is the first-round loser at $\pi([y x z])$. Then under the corresponding runoff rule, $y$ wins in $\pi([y x z])$ against $z$ at the second round. Hence, the truncation paradox occurs.

2. Necessity. Assume that the truncation paradox is liable to occur at $\pi$ in favor of $z$. By truncating their true preferences, members of some coalition, say $S$, favor the election of $z$ whom they strictly prefer to $x$. In the new voting situation $\pi^{\prime}, z$ wins the majority duel against $x$ or against $y$. First suppose that $z$ wins in $\pi^{\prime}$ against $x$ at the second round. Then $y$ is the first-round loser at $\pi^{\prime}$. Moreover, voters in $S$ all strictly prefer $z$ to $x$; and the truncation operation is intended, at the first round in $\pi^{\prime}$, to diminish the score of $y$. Thus the preference of each voter in $S$ is $z y x$. Hence $|S| \leq n_{z y x}(\pi)$. Therefore, in $\pi([z y x]), z$ also wins against $x$ and $y$ is the first round loser. Finally, suppose that $z$ wins in $\pi^{\prime}$ against $y$ at the second round. Then $x$ is the first-round loser at $\pi^{\prime}$. Voters in $S$ all strictly prefer $z$ to $y$; and the truncation operation is intended, at the first round in $\pi^{\prime}$, to diminish the score of $x$. The preference of each voter in $S$ is then $z x y$. This implies that $|S| \leq n_{z x y}(\pi)$. In $\pi([z y x]), z$ also wins against $y$ and $x$ is the first round loser.

Sufficiency. Assume that $z$ wins the majority duel against $y$ and $x$ is the firstround loser in $\pi([z x y])$. Then under the corresponding runoff rule, $z$ wins in $\pi([z x y])$ against $y$ at the second round. In the same way, suppose that $z$ wins the majority duel against $x$ and $y$ is the first-round loser in $\pi([z y x])$. Then under the corresponding runoff rule, $z$ wins in $\pi([z y x])$ against $x$ at the second round. In both cases, the truncation paradox occurs.

\section{Computations details for Proposition 4}

Given $0<\lambda \leq 1$, let $R_{x y}$ denote the set of all voting situations in which the truncation paradox is liable to occur in favor of some candidate $u$ under the runoff rule associated with the weight $\lambda$ while $x$ and $y$ are respectively the election winner and the challenger. Let $z$ be the first-round loser in each voting situation in $R_{x y}$. Denote by $R_{x y y}$ the subset of $R_{x y}$ that consists of all voting situations in which truncating preferences may favor the election of $y$; by $R_{x y z}$ the subset of $R_{x y}$ that consists of all voting situations in which truncating preferences may favor the election of $z$ against $x$ at the second round; and by $R_{x y z}^{\prime}$ the subset of $R_{x y}$ that consists of all voting situations at which truncating preferences may favor the election of $z$ against $y$ at the second round. Then by Proposition 3

$$
R_{a b}=R_{a b b} \cup R_{a b c} \cup R_{a b c}^{\prime} .
$$

Note that $R_{a b b}$ and $R_{a b c}$ are disjoint sets of voting situations since $y$ wins the majority duel against $z$ in each voting situation in $R_{a b b}$ while the converse holds in each voting situation in $R_{a b c}$. Therefore

$$
\left|R_{a b}\right|=\left|R_{a b b}\right|+\left|R_{a b c}\right|+\left|R_{a b c}^{\prime}\right|-\left|R_{a b b} \cap R_{a b c}^{\prime}\right|-\left|R_{a b c} \cap R_{a b c}^{\prime}\right| .
$$


Note that by Proposition 3, $R_{a b b}, R_{a b c}$ and $R_{a b c}^{\prime}$ are each defined by some set of linear constraints. Therefore the probability that the corresponding runoff rule exhibits the truncation paradox is derived by computing the volume of the polytopes $P_{a b b}, P_{a b c}$ and $P_{a b c}^{\prime}$ associated to $R_{a b b}, R_{a b c}$ and $R_{a b c}^{\prime}$ respectively. More precisely, by considering the six possible sets $R_{x y}$ for all two ordered pairs $(x, y)$ from $\{a, b, c\}$ and taking into account possible symmetries, the limit probability $P\left(F_{\lambda}, T P, I A C\right)$, under the IAC assumption, of observing a voting situation with three candidates in which the truncation paradox may occur is

$P_{\mathrm{TP}}\left(F^{\prime}\right)=720\left[\operatorname{vol}\left(P_{a b b}\right)+\operatorname{vol}\left(P_{a b c}\right)+\operatorname{vol}\left(P_{a b c}^{\prime}\right)-\operatorname{vol}\left(P_{a b b} \cap P_{a b c}^{\prime}\right)-\operatorname{vol}\left(P_{a b c} \cap P_{a b c}^{\prime}\right)\right]$

\section{E. Proof of Proposition 5}

Assume that preferences are single-peaked in such a way that candidate $c$ is bottom ranked by no voters. When candidate $a$ is elected, voters who prefer $b$ to $a$ are of type 4 or type 6 . But these voters do not affect the score of candidate $a$ by a sincere truncation of their preferences. Thus candidate $b$ cannot be elected by sincere truncation of preferences. Similarly, when candidate $b$ is elected, there is no way for voters who strictly prefer $a$ to $b$ to favor the election of $a$ by simply truncating their rankings. Therefore, the truncation paradox may only occur in favor of $c$ when $a($ or $b)$ is the winner of a one-shot scoring rule and preferences are single-peaked.

\section{F. Proof of Proposition 6}

i) Let us assume that candidate $a$ wins versus candidate $b^{5}$ with $\lambda \in\left[\frac{1}{2}, 1\right]$. Note we should have $S(\pi, \lambda, c) \leq S(\pi, \lambda, a)$ and $S(\pi, \lambda, c) \leq S(\pi, \lambda, b)$. It follows that

$$
S(\pi, \lambda, c)-\frac{S(\pi, \lambda, a)+S(\pi, \lambda, a)}{2}=\frac{2-\lambda}{2}\left(x_{5}+x_{6}\right)+\frac{2 \lambda-1}{2}\left(x_{4}+x_{2}\right) \leq 0 .
$$

This occurs if and only if $\lambda=\frac{1}{2}$ and $x_{5}=x_{6}=0$. In this case, the three candidates all tie and there is no route for the profitable truncation of preferences.

Now suppose that $\lambda \in] 0, \frac{1}{2}$ [ and show that the paradox can occur for all $\lambda$ in this interval. Let us assume a voting situation where $n_{2}=n_{5}=\alpha$ and $n_{3}=$ $n_{4}+1=z$ with $z=\left\lceil\frac{2}{\lambda}-3\right\rceil+1$ and $\alpha=\left\lfloor\frac{2 z-1}{1-2 \lambda}\right\rfloor-1$ for $\left.\lambda \in\right] 0, \frac{1}{2}[$. The scores are: $S(\pi, \lambda, a)=\alpha+\lambda z, S(\pi, \lambda, b)=\alpha+\lambda(z-1)$ and $S(\pi, \lambda, c)=2 z-1+2 \lambda \alpha$. It follows that $S(\pi, \lambda, a)>S(\pi, \lambda, b), S(\pi, \lambda, b)>S(\pi, \lambda, c)$. Candidate $c$ is eliminated and candidate $a$ wins the second run since $a M(\pi) b$. Assume that all the voters

${ }_{5}^{5}$ The symmetric to the case "candidate $b$ wins the second stage versus $a$ " is handled in a similar way. 
of type 3 truncate. The new scores are: $S\left(\pi^{\prime}, \lambda, a\right)=\alpha, S\left(\pi^{\prime}, \lambda, b\right)=S(\pi, \lambda, b)$ and $S\left(\pi^{\prime}, \lambda, c\right)=S(\pi, \lambda, c)$. We still have $S(\pi, \lambda, b)>S(\pi, \lambda, c)$. Let us show that $S(\pi, \lambda, c)-S\left(\pi^{\prime}, \lambda, a\right)$.

$$
\begin{aligned}
S(\pi, \lambda, c)-S\left(\pi^{\prime}, \lambda, a\right) & =2 z-1-(1-2 \lambda) x \\
& =2 z-1-(1-2 \lambda)\left(\left\lfloor\frac{2 z-1}{1-2 \lambda}\right\rfloor-1\right) \\
& =2 z-1-(1-2 \lambda)\left\lfloor\frac{2 z-1}{1-2 \lambda}\right\rfloor+(1-2 \lambda)
\end{aligned}
$$

For all $\lambda \in] 0, \frac{1}{2}[$, we have $z>1$; so, $2 z>1$ and $2 z-1>0$. Also, $(1-2 \lambda)>0$ and we know that $(1-2 \lambda)\left\lfloor\frac{2 z-1}{1-2 \lambda}\right\rfloor \leq 2 z-1$. Thus, $S(\pi, \lambda, c)-S\left(\pi^{\prime}, \lambda, a\right)>0$. So, candidate $a$ is eliminated. Since $x+2 z-1>\alpha, c M\left(\pi^{\prime}\right) b$ : candidate $c$ is the new winner. Thus, by sincere truncation of their rankings, voters of type 3 have favored their best candidate.

ii) Assume that candidate $a$ wins the second stage versus $c .{ }^{6}$ This means that $a M c$ through $x_{2} \geq x_{4}+x_{5}+x_{6}$ and thus $x_{2} \geq \frac{1}{2}$. Note that only voters of type 5 have an incentive to manipulate and the possibility to affect the score of $a$ by sincere truncation of their rankings in favor of $c$. But by any truncation from $\pi$ to a new voting situation $\pi^{\prime}$, we still have $S\left(\pi^{\prime}, \lambda, a\right) \geq \frac{1}{2}$ and $S(\pi, \lambda, b)=x_{4}+\lambda x_{6} \leq$ $x_{4}+x_{5}+x_{6} \leq \frac{1}{2}$. Therefore candidate $c$ still gets through to the second round and wins the election against $c$.

iii) Let us assume that candidate $c$ wins the second stage versus $a$. Let us first consider $\lambda=1$. Evidently, candidate $c$ wins. If she wins versus candidate $a$, this means that $S(\pi, \lambda, a)>S(\pi, \lambda, b)$ which is equivalent to (i) $n_{2}+n_{5}>n_{4}+n_{6}$. Candidate $b$ will become the new winner after voters of type 4 truncate if (ii) $S(\pi, \lambda, b)>S\left(\pi^{\prime}, \lambda, c\right)$; and that (iii) $b M\left(\pi^{\prime}\right) a$. This last requirement is equivalent to $n_{4}+n_{6}>n_{2}+n_{5}$ which contradicts (i): voters of type 4 cannot manipulate for $\lambda=1$. If voters of type 2 truncate, nothing will happen since the new score of candidate $c$, although diminished by $\lambda n_{2}$, will still be greater than that of candidate $b$ : voters of type 2 cannot manipulate for $\lambda=1$. Thus, the truncation paradox is not possible for $\lambda=1$. To prove that it can happen for $\lambda \in] 01[$, one can consider a profile such that $n_{2}=3, n_{4}=2 n_{5}=1$ and $n_{6}=1$.

\section{References}

Brams, S.J. (1982) The AMS nominating system is vulnerable to truncation of preferences. Notices of the American Mathematical Society 29, 136-138.

Brandt, F., Hofbauer, J., \& Strobel, M. (2018) Exploring the no-show paradox for Condorcet extensions using Ehrhart theory and computer simulations. Working paper.

${ }^{6}$ This is symmetric to the case "candidate $b$ wins the second stage versus $c$ ". 
Bruns, W., \& Ichim, B. (2010) Normaliz, algorithms for affine monoids and rational cones. Journal of Algebra 324(5), 1098-1113

Bruns, W., Ichim, B., \& Söger, C. (2019) Computations of volumes and Ehrhart series in four candidates elections. Forthcoming in Annals of Operations Research, https://doi.org/10.1007/s1047

Bruns, W., Ichim, B., Römer, T., Sieg, R., \& Söger, C. (2018) Normaliz: Algorithms for rational cones and affine monoids. Working paper available at http://normaliz.uos.de.

Büeler, B., Enge, A., \& Fukuda, K. (2000) Exact volume computation for polytopes: a practical study. In Polytopes-combinatorics and computation, Kalai, G., \& Ziegler G.M. (Eds.), Pages 131-154, Birkhäuser, Basel.

Cervone, D., Gehrlein, W.V., \& Zwicker, W. (2005) Which scoring rule maximizes Condorcet efficiency under IAC? Theory and Decision 58, 145-185.

Diss, M., \& Gehrlein, W.V. (2015) The true impact of voting rule selection on Condorcet efficiency. Economics Bulletin 35(4), 2418-2426.

Diss, M., \& Gehrlein, W.V. (2012) Borda's paradox with weighted scoring rules. Social Choice and Welfare 38, 121-136.

Doron, G., \& Kronick, R. (1977) Single Transferable Vote: An example of a Oerverse Social Choice Function. American Journal of Political Science 21(2), 303-311.

Duddy, C. (2013) Condorcet's principle and the strong no-show paradoxes. Theory and Decision 77(2), 275-285.

Felsenthal, D.S. (2012). Review of Paradoxes Afflicting Procedures for Electing a Single Candidate in Electoral Systems : Paradoxes, Assumptions, and Procedures, Studies in Choice and Welfare, Felsenthal, DS, \& Machover, M. (Eds.), Springer Berlin Heidelberg.

Fishburn, P.C., \& Brams, S.J. (1983) Paradoxes of preferential voting. Mathematics Magazine 56, 207-214.

Fishburn, P.C., \& Brams, S.J. (1984) Manipulability of voting by sincere truncation of preferences. Public Choice 44, 397-410.

Gehrlein, W.V., \& Fishburn, P.C. (1976) The probability of the paradox of voting: A computable solution. Journal of Economic Theory 13, 14-25.

Gehrlein, W.V, \& Lepelley, D. (2017) Elections, Voting Rules and Paradoxical Outcomes. Publisher, Springer-Verlag.

Gehrlein, W.V, \& Lepelley, D. (2011) Voting paradoxes and group coherence. Springer, Berlin/Heidelberg.

Gehrlein, W.V., Lepelley, D., \& Moyouwou, I. (2015) Voters preference diversity, concepts of agreement and Condorcet's paradox. Quality and Quantity 49 (6), 2345-2368.

Jimeno, J.L., Pérez, J., \& García, E. (2009). An extension of the Moulin No Show Paradox for voting correspondences. Social Choice and Welfare 33, 343-359.

Kamwa, E.(2019) On the Likelihood of the Borda Effect, The Overall Probabilities for General Weighted Scoring Rules and Scoring Runoff Rules. Group Decision and Negotiation 28(3), 519-541 . 
Kamwa, E., Merlin, V., \& Top, F. (2018) Scoring Runoff Rules, Single-peaked Preferences and Paradoxes of Variable Electorate. Working paper, CREM University of Caen.

Kamwa E., \& Valognes, F.(2017) Scoring rules and preference restrictions: The strong Borda paradox revisited. Revue d'Economie Politique 127(3), 375-395.

Lawrence, J. (1991) Polytope volume computation. Mathematics of Computation 57(195), 259-271.

Kuga, K., \& Hiroaki, N. (1974) Voter antagonism and the paradox of voting. Econometrica 42(6), 1045-1067

Lepelley, D., Chantreuil, F., \& Berg S. (1996) The likelihood of monotonicity paradoxes in runoff elections. Mathematical Social Sciences 31(3), 133-146.

Lepelley, D., El Ouafdi, A., \& Smaoui, H. (2018). Probabilities of electoral outcomes in four-candidate elections. Preprint available at https://doi.org/10.13140/RG.2.2.27775.87201.

Lepelley, D., Louichi, A., \& Smaoui, H. (2008) On Ehrhart polynomials and probability calculations in voting theory. Social Choice and Welfare 30, 363-383.

Lepelley, D., \& Mbih, B. (1996) The vulnerability of four social choice functions to coalitional manipulation of preferences. Social Choice and Welfare 11, 253-265.

Lepelley, D., \& Merlin, V. (2001) Scoring runoff paradoxes for variable electorates. Economic Theory 14(1), 53-80.

Mbih, B., Courtin, S., \& Moyouwou, I. (1996) Susceptibility to coalitional strategic sponsoring The case of parliamentary agendas. Public Choice 144, 133-151.

Moulin, H. (1988) Condorcet's principle implies the no show paradox. Journal of Economic Theory 45(1), 53-64.

Moyouwou, I., \& Tchantcho, H. (2015) Asymptotic vulnerability of positional voting rules to coalitional manipulation. Mathematical Social Sciences 89, 70-82.

Nurmi H. (1999) Voting Paradoxes and How to Deal with Them. Springer-Verlag.

Plassmann, F. \& Tideman, T.N. (2013) How frequently do different voting rules encounter voting paradoxes in three-candidate elections? Social Choice and Welfare 42(1), 31-75.

Pritchard, G., \& Wilson, M. (2007) Exact results on manipulability of positional voting rules. Social Choice and Welfare 29, 487-513.

Smith, J.H. (1973). Aggregation of preferences with variable. Econometrica 41, 1027-1041. 